\title{
Design of a RESTful Web information system for drug prescription and administration
}

\author{
Lorenzo Bianchi, Federica Paganelli, Member, IEEE, Maria Chiara Pettenati, Stefano Turchi, Lucia \\ Ciofi, Ernesto Iadanza, Member, IEEE, Dino Giuli, Senior Member, IEEE
}

\begin{abstract}
Drug prescription and administration processes strongly impact on the occurrence of risks in medical settings for they can be sources of Adverse Drug Events (ADEs). A properly engineered use of Information and Communication Technologies (ICT) has proven to be a promising approach to reduce these risks. In this work, we propose PHARMA, a Web information system which supports healthcare staff in the secure cooperative execution of drug prescription, transcription and registration tasks. PHARMA allows the easy sharing and management of documents containing drug-related information (i.e., drug prescriptions, medical reports, screening), which is often inconsistent and scattered across different information systems and heterogeneous organization domains (e.g., departments, other hospital facilities). PHARMA enables users to access such information in a consistent and secure way, through the adoption of REST and web-oriented design paradigms and protocols. We describe the implementation of the PHARMA prototype and we discuss the results of the usability evaluation that we carried out with the staff of a hospital in Florence (Italy).
\end{abstract}

Index Terms - CPOE, Distributed information systems, Drug prescriptions, Drug Administration, Resource Oriented Architecture, REST, Web Services.

\section{INTRODUCTION}

$\mathrm{S}_{\mathrm{d}}^{\mathrm{E}}$ EVERAL challenges in healthcare require further developments in Information and Communication Technologies. Indeed, issues such as usability, cooperation, security, interoperability and consistency of data can directly or indirectly affect patient safety. One important kind of error in medicine is Adverse Drug Events (ADE). An ADE is defined as "an injury resulting from the use of a drug". Under this definition, the term ADE includes harm caused by the drug (adverse drug reactions and overdoses) and harm from the use of the drug (including dose reductions and discontinuation of drug therapy)" [1].

Manuscript received December 27, 2012; revised April 19, 2013; accepted September 13, 2013

L. Bianchi is with the Department of Information Engineering, University of Florence, 50139, Italy (e-mail: lorenzo.bianchi@unifi.it).

F. Paganelli is with the National Interuniversity Consortium for Telecommunications, at the Research Unit of the University of Florence, 50139, Italy (phone: +39 055 4796382; fax: +39 055 4796427; e-mail: federica.paganelli@unifi.it).

M.C. Pettenati is with the ICON Foundation, Sesto Fiorentino, Italy (e-mail: mc.pettenati@iconfoundation.net).

S. Turchi, L. Ciofi, and E. Iadanza are with the Department of Information Engineering, University of Florence, 50139, Italy (e-mail: lucia.ciofi, stefano.turchi, ernesto.iadanza @ unifi.it).
Drug prescription and administration processes can also become sources of high ADE risks. The use of ICT is considered a promising approach to reduce these risks. To this end, several Computerized Physician Order Entry (CPOE) systems have been proposed and tested in hospital environments. CPOE refers to a variety of computer-based systems that offer services for automating the medication ordering process. Current available approaches often lack in user friendliness, integration with existing systems and devices, workflow and collaborative tasks support, thus reducing or worse - hindering the ADE reduction goal for which they were introduced.

Our approach consists in the development of a Web information system called PHARMA, designed to support the healthcare staff in the secure cooperative execution of drug prescription, transcription and registration tasks. PHARMA allows the easy sharing and management of documents containing drug-related information (i.e., drug prescriptions, medical reports, screening), which is typically distributed across different information systems and heterogeneous organization domains (e.g., departments, other hospital facilities). PHARMA enables users to access such information in a consistent and secure way, through the adoption of REST and web-oriented design paradigms and protocols.

This manuscript is structured as follows: Section II introduces the problem of ADE and the impact of ICT on the risk mitigation, while Section III discusses related work in CPOE systems. Section IV describes the design of the proposed PHARMA system. In Section V we describe the implementation of the first prototype and in Section VI we discuss preliminary validation results. Section VII concludes the manuscript.

\section{BACKGROUND}

ADE can occur because of prescription, transcription, preparation, distribution and/or administration errors. Prescription and transcription errors represent more than half of all the ADE, thus impacting in societal costs that can become unsustainable [2][3][4][5]. Studies performed in the US showed that a significant percentage (between $7 \%$ and $16 \%$ ca.) of fatal incidents that resulted from medical errors, were drugs-related $\mathrm{ADE}$, for an estimated total cost of 1.56-5.6 billion dollars per year [6].

Approaches to ADE reduction are of worldwide interest: the British Department of Health recommends the wider use of 
electronic prescription systems to reduce the risk of medication errors [7] and the Italian Health Ministry in 2003 constituted a Technical Risk Reduction Board that issued several recommendations on this topic [8]. The electronic prescription (or e-prescription) is supported by electronic systems called Computerized Physician Order Entry (CPOE). A basic CPOE system ensures standardized, legible, complete orders by only accepting typed orders in a standard and complete format. Many CPOE systems include or interface with clinical decision support systems (CDSS) [9].

Several studies analyzed the benefits and the risks related to the adoption of ICT solutions (especially CPOE and CDSS systems) to improve patient safety [10].

According to a systematic review analysing the relative risk reduction on medication errors and ADE caused by CPOE [7], 23 out of 25 studies on the effects of the medication error rate showed a significant relative risk reduction (13\% to $99 \%$ ). Six out of nine studies that analysed the effects on potential ADE showed a significant relative risk reduction (35\% to $98 \%)$. Four out of seven studies that analysed the effect on ADE showed a significant relative risk reduction ( $30 \%$ to $84 \%$ ), concluding that electronic prescription can reduce the risk of medication errors and ADE. Moreover, some studies [11][12] showed that the use of CPOE systems in a hospital can help to reduce drug prescription errors up to $80 \%$. Other studies indicate that the use of a CPOE leads to an improvement in medical treatments with a relative percentage reduction of prescription errors of up to 95\% [7][13][14]. Other studies found that CPOEs and CDSSs can substantially reduce medication error rates, although most of these studies did not highlight differences in diverse types of adverse drug events and often evaluated small numbers of locally developed pilots or prototypal systems [15][16].

On the other hand, several studies also conclude that CPOE could even introduce new risks that must be taken into account. Koppel et al. [17] claim that a leading CPOE system often facilitated medication error risks. As CPOE systems are implemented, clinicians and hospitals must attend to errors that these systems may cause in addition to errors they prevent. Nebeker et al. [18] showed that high rates of ADEs may continue to occur after implementation of CPOE and related computerized medication systems which lack decision support for drug selection, dosing, and monitoring.

These findings demonstrate that, in designing any technological system aimed at reducing errors and ADE, it is critical to start from a deep process analysis and a rigorous risk assessment [18][19].

\section{RELATED WORK}

This section presents related work in the design and development of ICT systems for drug prescriptions management in hospital settings.

CureCPOE [20] offers several features such as prescription management, patient-centered decision support, patient safety with real time patient identification, drug dose recommendations. The system has been developed by leveraging the Windows DNA technologies for distributed application development.

The PatientKeeper CPOE [21] supports the implementation of physician workflows, including the insertion of order sets in the system and their seamless distribution across the appropriate departments within the hospital. However, the approach that was adopted for interoperating with existing hospital information systems is not documented.

Del Fiol et al. [22] propose an XML-based model enabling the clinical experts to develop order sets without the support of the IT technical staff. Thus, they focus on the design of an authoring and management tool but they do not provide details on the interoperability with external systems.

Hsieh et al. [23] propose the Mobile Electronic Medication Administration Record (ME-MAR) system, which provides the nursing staff with services for drugs administration. ME-MAR leverages service-oriented design principles and is compliant with the HL7 standard [24]. However, the system does not support physicians in drug prescription tasks.

An agent-based approach is proposed in [25] to face the problem of integrating CDSS and CPOE systems in the clinical workflow management system. The proposed system is at a preliminary stage of design.

\section{A. Motivation of our work}

Identifying and understanding the types and the causes of unintended adverse consequences associated with CPOE will help us to design a system able to reduce the clinical risk for patients, while minimizing many side-effects such as more/new work for clinicians, legal problems related to paper persistence, and untoward changes in communication practices [26].

To support the CPOE diffusion within healthcare scenarios along with the preservation of the patients' safety, some guidelines have been specified [27][28]. Recommendations for a CPOE implementation have been proposed by the Physician Order Entry Team (POET), of the Oregon Health \& Science University [29]. This analysis of recommendations for a successful CPOE implementation highlights the importance of some crucial factors to reduce the unpredictable problems caused by CPOE misuses [30]:

1) User friendliness is very important, since the more difficult a system is to be used, the less it is likely to be adopted. In addition, a system with a complex interface is more likely to be error-prone.

2) A CPOE should support integration with different systems and devices so as not to alter the usual practitioner workflow.

3) A CPOE should support collaborative work, because different professionals (medical teams, technicians and administrative personnel) usually work around a patient at the same time. Considering the variety of professional figures that use the CPOE, it is important to be very careful about the terminology implemented in the system to enforce understandability.

4) Time is a significant factor: exploitation of IT systems for health support should not increase the time required for 
medical procedures.

5) Finally, a determinant factor is the training of the personnel. It is very important to guarantee CPOE users continuous support so as to achieve an optimal use of the system. Indeed, Ozdas and Miller [30] highlight the fact that potential CPOE users are often discouraged by the effort required to learn the new technology.

Our work aims primarily at addressing requirements 1 ), 2), and 3) by proposing PHARMA, a web information system that supports the healthcare staff in the secure cooperative execution of drug prescription, transcription and registration tasks. Since these tasks are error-prone, PHARMA can help in minimizing prescription and transcription errors. Interoperability with external systems is addressed through the adoption of web design principles and standard specifications, i.e., the Representational State Transfer (REST) paradigm. User friendliness is addressed through the adoption of technical standards for software usability.

By pursuing these three objectives, we also aim at addressing the main limitations of related work. As mentioned above, Bouzguenda and Turki [25] provide only preliminary design details; other systems [21][22] do not clearly address issues of interoperability with external systems; the work in [23] offers limited support to cooperation since it is addressed to the nurse staff, but not to physicians.

The achievement of requirements 4) and 5) is not an objective of this research and it will be tackled in the future through tests in specific hospital environments.

\section{PHARMA}

PHARMA is a web information system aimed at supporting the health care staff in carrying out drug prescription, transcription and registration tasks.

PHARMA offers the following services:

- Clinical Information Retrieval: physicians and nurses can search and retrieve patient's clinical data, (e.g., medical records, daily therapies, and medical reports).

- Medical Record Management: health care staff can create new medical records. Patient medical records typically contain heterogeneous types of information (e.g., clinical record, consultations, diagnoses). In this paper we especially focus on medical reports and daily therapies.

- Medical Report Management: physicians can access and update a patient medical report.

- Daily Therapies Management: physicians can create new daily therapies (e.g., drug prescriptions) for a given patient; nurses can access the prescribed therapies and modify specific records (e.g., registration of drug administration). The design of the daily therapies management interface was inspired by the Integrated Therapeutic Chart (ITC) specifications published by Tuscany Region.

This system is designed to enable cooperation amongst hospital personnel by sharing clinical information that is relevant to the drug prescription and administration phases. This information is typically distributed across multiple information systems. For example, in a basic but significant scenario, clinical data may be distributed across two or more systems pertaining to different organization domains (e.g. different departments). When a physician accesses a patient's medical record, he needs to retrieve all the patient's information. For this reason, a tool that aggregates all the information distributed across various departments can help in generating a complete clinical picture. According to the organization roles, users can access and modify different information fields.

PHARMA is capable of ensuring that information is handled in a consistent and secured way. To achieve these objectives, our approach consists of:

1) Representing the information entities that have to be handled during the drug prescription, transcription and registration tasks by exploiting a structured and graph-based information model. As described in the following sections, this model allows to easily design, compose and handle information nodes that are gathered from heterogeneous legacy information systems.

2) Exposing these information nodes as web resources that can be accessed through a uniform interface. This is achieved through the adoption of web principles (i.e., REST architectural style) and protocols (e.g. HTTP, HTTPS).

In this way, patient information can be represented as a graph of web-addressable URIs. End users can access and modify these URI-addressable information nodes and see actions that have previously been performed by other colleagues on those nodes. In this sense, the degree of supported collaboration depends on the depth of granularity applied in the design of the information graph.

This approach has been implemented by leveraging the capabilities offered by the InterDataNet (IDN) middleware. IDN is an open source middleware, developed in our laboratory [31], that offers capabilities for representing information units and their structural and semantic relations on the Web, and making them accessible through a RESTful uniform interface.

\section{A. REST guidelines}

The REST paradigm was proposed by Roy Fielding in his doctoral dissertation [32] as an architectural style for building large-scale distributed and cooperative hypermedia systems.

REST relies on the main concept of "resource", which is an abstract information entity. On the REST vision, data sets and objects handled by client-server application logic are modeled as resources. Resources are identified via a URI and can be manipulated through a uniform interface. REST is not tightly bound to any specific protocol, even though HTTP is widely adopted for its implementation.

REST key principles are [32]:

1) Use URIs to identify resources. The resources are exposed by servers through URIs. URIs belong to a global addressing space, hence resources identified with URIs have a global scope.

2) Adopt a uniform interface. The interaction with the 
resource is fully expressed with four primitives: create, read, update and delete. In HTTP they are mapped on the PUT, GET, POST and DELETE verbs: GET gets the resource state; PUT sets the resource state; DELETE deletes a resource; POST extends a resource by creating a child resource [33].

3) Adopt self-descriptive messages. Each message contains the information required for its management. Metadata is used for content negotiation, errors notification, etc.

4) Adopt stateless interactions. Each request from a client to a server must contain all the information needed to understand the request. Session state is kept by the client and no client session data are stored in the server. Instead, the server manages and stores the state of the resources.

A widely adopted methodology for RESTful service design is described in [34]. Firstly, the designer has to model the set of information and objects on which the service will operate, and identify a set of resources which properly represents such a domain. Then, for each resource the designer has to:

- name the resource using a URI;

- identify the subset of allowed operations on that resource;

- design the representation(s) of the resource as accepted by the client (in a request) and sent to the client in a response;

- connect the resource with other resources through hyperlinks;

- analyze the typical course of events by considering how the service is supposed to behave and possible error conditions.

In some cases, an operation on a resource (i.e., a RESTful service) can be executed by invoking a set of operations on the resource or on different resources [35]. These types of operations are typically referred to as "composite RESTful services". By specifying composition patterns for RESTful services, we can explicitly model the relations among distributed information resources.

\section{B. InterDataNet middleware}

InterDataNet (IDN) [36] is an open source middleware offering capabilities for representing and managing information units and their structural and semantic relations on the Web, in a RESTful way. For the sake of conciseness, here we provide a brief introduction of IDN, further details can be found in [36] and [37].

The main goal of IDN is to enable the easy employment and reuse of globally web-addressable information units to support collaboration around data. For this purpose, IDN considers documents as first class entities. A document can be defined as "a set of information pertaining to a topic, structured for human comprehension, represented by a variety of symbols..." [38]. Humans are thus very comfortable conceiving data aggregation in documental form. Therefore, in IDN, documents represent a structured aggregation of data that conveys some meaningful (and shared) information in a given application domain. In the following, we refer to a document in IDN as IDN-Document. The IDN-Information Model (IDN-IM) defines the rules for data organization while building an IDN-Document.
More precisely, an IDN-Document is a graph $\mathrm{G}=(\mathrm{V}, \mathrm{E})$ where $\mathrm{V}$ is the set of vertices and $\mathrm{E}$ is the set of edges. The elements of $\mathrm{V}$ and $\mathrm{E}$ are the nodes containing the granular information (IDN-Nodes) and the relations between IDN-Nodes, respectively. IDN supports different types of relations between IDN-Nodes such as containment and reference. The containment relation is expressed using the Aggregation Link, which is a directed edge which starts from the container element and points to the content element. Similarly, the reference relation is expressed using the Reference Link which is a directed edge which starts from the referring element and points to the referred element.

Through the IDN-IM it is possible to define an IDN-Document as an aggregation of data provided by different information sources. Indeed, an IDN-Node can be linked to by more than one IDN-Document, thus favoring information reuse across different applications. This is possible since each IDN-Node is associated with the information provider that is authoritative for the information to which the IDN-Node links.

Hence, gathering information from the proper sources enforces an appropriate responsibility distribution across the information providers, who are responsible for the quality of the information provided. Figure 1 shows the concept of information reuse between IDN-Documents belonging to different domains (represented as space regions surrounded by dotted curves). In the example, the IDN-Document $\mathrm{D}$ is aggregated by the IDN-Documents A and B.

In addition, the IDN-IM can be extended with metadata enforcing licensing, provenance, consistency, versioning and availability properties attached to IDN-Nodes and affecting IDN-Documents [36]. Such features are crucial to support effective and trusted collaboration in real world scenarios.

IDN-Documents are exposed as resources by the IDN-Service Architecture (IDN-SA) middleware, which provides services for accessing and managing IDN-native information as well as information gathered from heterogeneous legacy systems through proper adapters.

The IDN-Service Architecture exposes a set of REST APIs for addressing, resolving and handling information represented as IDN-Documents. It also offers a set of horizontal services, including names resolution and security management. By exploiting these REST APIs, it is possible to develop web applications (called IDN-Compliant Applications) that browse

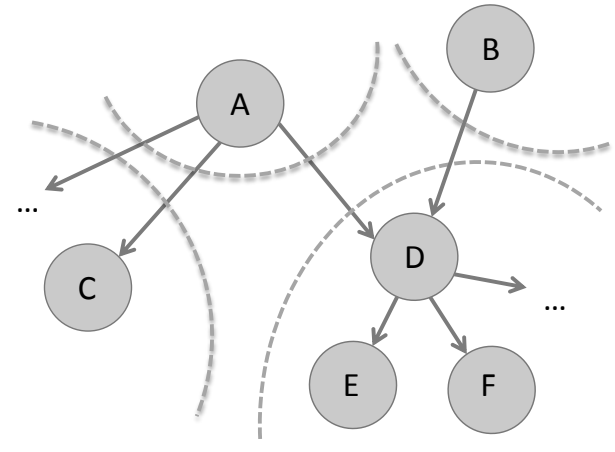

Fig. 1. Interlinked IDN-Documents across different domains. 
and handle graphs of distributed URI-addressable information units (i.e. IDN-Documents). In the following section we discuss how REST principles and the IDN model and middleware have been exploited in the design of PHARMA.

\section{Resource-oriented design of PHARMA}

As previously mentioned, the first step in resource-oriented system design is the identification of resources. We thus identified a set of resources that are relevant to the drug prescription and administration processes (see the entity names in Table I).

In this phase, we took as reference the model of the Integrated Therapeutic Chart (ITC) [39]. The ITC is a form for drug prescription, preparation and administration that has been defined and recommended by the Tuscany Region to reduce transcription and misunderstandings between clinicians, pharmacists and nurses by fully documenting the therapeutic process. The paper-based ITC version has been adopted in the 36 acute care hospitals in Tuscany (representing more than $80 \%$ of the hospitalization structures in Tuscany) [39].

Its design was directed towards the specification and enforcement of drug administration workflows identifying and checking six main conditions: 1) the right person;2) the right drug; 3) the right quantity; 4) the right administration way; 5) the right time; 6) the right registration [40]. The ITC enforces the accountability for the prescription and administration phases, by tracking and logging all the operations made by the health care staff, and helps in reducing transcription errors that may occur when medical prescriptions are manually transcribed and duplicated in other documents.

We adopted the ITC to model the daily therapies. In addition, we used the International Classification of Diseases (ICD) [41] to code medications.

According to the RESTful design methodology, the next step consists of specifying relations among resources. Figure 2 shows the main relations between the identified resources. Since we chose to adopt the IDN middleware, the relations can be either reference or aggregation links, according to the definitions provided in subsection IV.B. For example, a

TABLE I

HTTP OPERATIONS.

\begin{tabular}{rcccc}
\hline \hline Entity Name & GET & PUT & POST & DELETE \\
\hline Hospital Files Manager & yes & no & yes & no \\
Hospital File & yes & yes & no & no \\
Medical Records & yes & yes & no & no \\
Medical Records Box & no & no & yes & no \\
Medical Records Manager & no & no & yes & no \\
Medical Record & yes & yes & no & no \\
Daily Therapies & yes & yes & no & no \\
Daily Therapies Box & no & no & yes & no \\
Daily Therapy & yes & yes & no & no \\
Consent & yes & yes & no & no \\
Reports Box & no & no & yes & no \\
Reports Manager & no & no & yes & no \\
Report & yes & yes & no & yes \\
\hline \hline
\end{tabular}

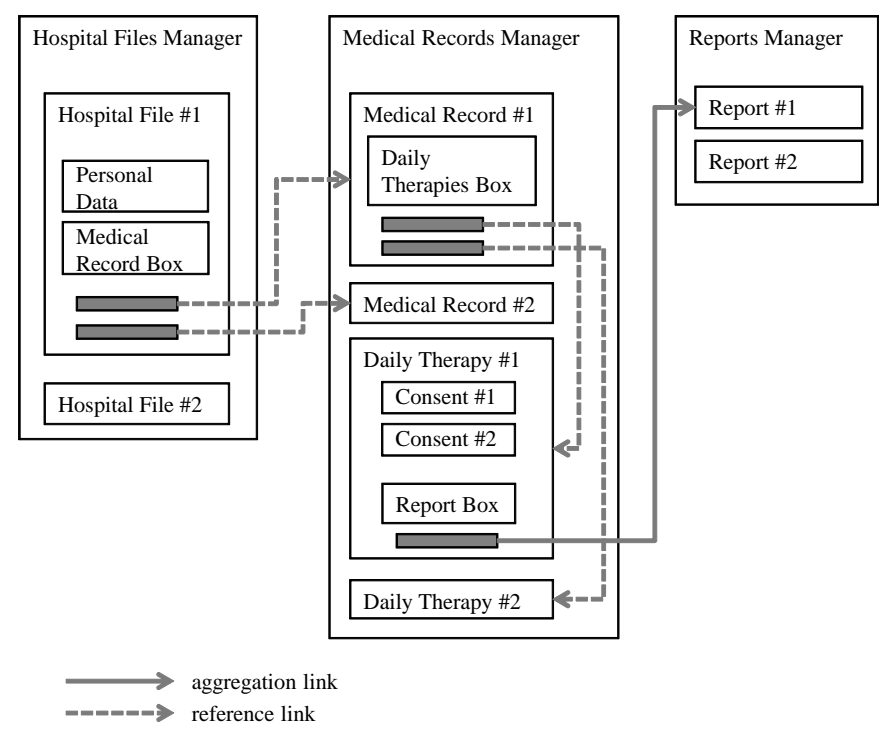

Fig. 2. Relations among resources.

patient's Hospital File (Hospital File \#1) is modeled as a resource containing reference links to the Medical Records related to that patient (e.g., Medical Record \#1 and \#2). Each Medical Record contains reference links pointing to various documents, such as Daily Therapies.

The REST design provides the definition of nice URIs for locating and addressing resources. The main resources have the URIs listed in Table II. The adoption of alias URIs in the PHARMA model design enables the addressability of the single resource via multiple paths. For example, URI 6 and URI 7 in Table II identify a unique resource that can be accessed through multiple paths. For the sake of understandability it is convenient to express the hierarchy among entities in the URI path.

The semantics of REST operations on a given resource is defined. Table I shows the REST operations that are allowed on PHARMA resources. For example, a Daily Therapy can be read, created, modified but not deleted: indeed, the allowed HTTP requests are GET for reading and PUT for creating and editing it. Users can invoke a subset of these operations, depending on their role (e.g., a new prescription can be added only by a physician).

The medical records box, daily therapies box, and reports box resources have been specified as composite services. An operation on these resources is translated into a workflow, i.e. a

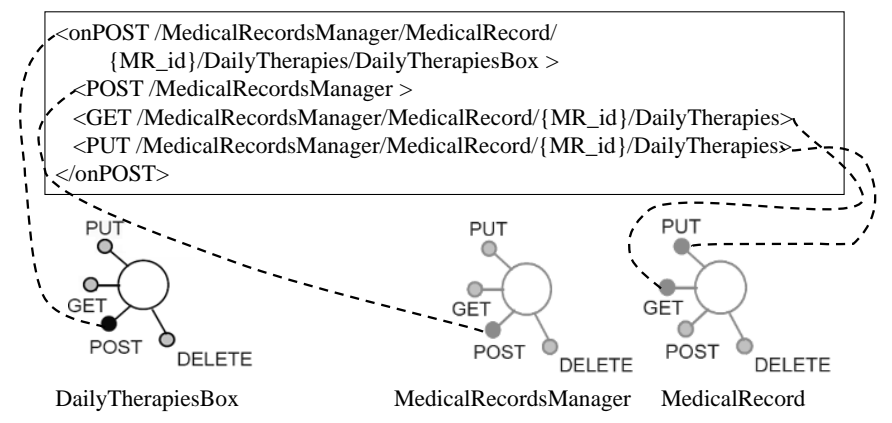

Fig. 3. Example of composed RESTful service. 
flow of invocations on other resources. Figure 3 shows an HTTP POST operation on the Daily Therapies Box resource that creates an association between a Medical Record and a newly created Daily Therapy. This phase is accomplished by invoking a sequence of operations: i) a POST operation on the Medical Record Manager resource is invoked to create a new Daily Therapy; ii) the representation of the current Medical Record resource is retrieved through a GET operation; and iii) the state of the Medical Record is modified through a PUT operation that adds the reference link to the new Daily Therapy.

The next step in the REST methodology is the design of the resources' representation. To this end, we exploited the Information Model (IDN-IM).

In other words, PHARMA leverages the IDN-Document formalism for representing information. Figure 4 shows a detail of the IDN-IM that represents a set of resources, including a Daily Therapy, a Drug Prescription and a Schedule. The vertices are the information entities and the edges are aggregation and reference links. It also shows the granular detail of the Daily Therapy including the Drug Prescription representation (identified by the URI../Prescr $/\left\{\mathrm{P}_{-} \mathrm{id}\right\}$ ). Each prescription is composed of one or more Schedule vertices. The Schedule vertex is related to drug administration information, such as the administration time and the name of the operator performing the administration task. Furthermore, a Daily Therapy could include a specific consent form before passing to the drug administration phase.

The last step in the REST methodology is the specification of use cases describing how the system is supposed to behave. We carried out an in-depth use-case analysis by adopting the BPMN language (Business Process Model and Notation) [42] and the BPMN Process Modeler [43] software developed by BizAgi. Detailed results are reported in [44].

Figure 5 shows how a new drug prescription use case is carried out through a sequence of REST invocations. A physician willing to create a new daily therapy and, consequently, a new drug prescription, interacts with the system using a web client and performs four actions: 1) retrieves the patient's medical history, 2) retrieves the patient's medical record, 3) creates a new daily therapy document and 4) modifies the created patient's daily therapy by adding a new drug prescription. Figure 5 shows these steps in detail. The resources delivered to the client are IDN-Documents. In the first step, the returned IDN-Document contains a representation of the patient's hospital file. The client extracts from the hospital file the links which refer to medical records and selects the one related to the appropriate medical record. In the second step, the

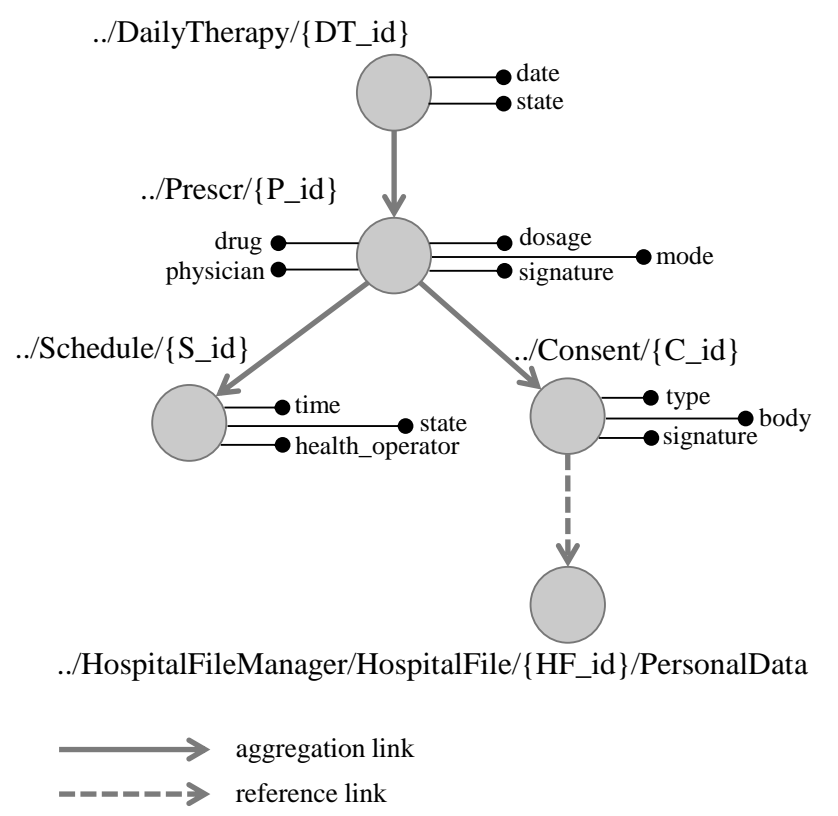

Fig. 4. A detail of the Information Model. Daily therapy (as a part of a Medical Record).

TABLE II

URIS USED IN THE SYSTEM

\begin{tabular}{|c|c|c|}
\hline No. & Resource Name - URI & Type \\
\hline 1 & /HospitalFilesManager & canonical \\
\hline 2 & /MedicalRecordsManager & canonical \\
\hline 3 & /ReportsManager & canonical \\
\hline 4 & /HospitalFilesManager/HospitalFile/\{HF_id $\}$ & canonical \\
\hline 5 & /HospitalFilesManager/HospitalFile/\{HF_id $\} /$ MedicalRecords & canonical \\
\hline 6 & /MedicalRecordsManager/MedicalRecord/\{MR_id $\}$ & canonical \\
\hline 7 & /HospitalFilesManager/HospitalFile/\{HF_id\}/MedicalRecord/\{MR_id\} & alias of 6 \\
\hline 8 & /MedicalRecordsManager/MedicalRecord/\{MR_id\}/DailyTherapies & canonical \\
\hline 9 & /MedicalRecordsManager/DailyTherapy/\{DT_id\} & canonical \\
\hline 10 & /MedicalRecordsManager/MedicalRecord/\{MR_id $\} /$ DailyTherapies $/\left\{D T \_i d\right\}$ & alias of 9 \\
\hline 11 & /ReportsManager/Report $/\left\{R \_i d\right\}$ & canonical \\
\hline 12 & $/$ MedicalRecordsManager/DailyTherapy//DTT_id $\} / T r e a t m /\left\{T \_i d\right\} / R e p o r t /\left\{R \_i d\right\}$ & alias of 11 \\
\hline 13 & /MedicalRecordsManager/DailyTherapy/\{DT_id $\} /$ Treatm//T_id $\} /$ Consent $/\left\{C \_i d\right\}$ & canonical \\
\hline 14 & $/$ MedicalRecordsManager/DailyTherapy//[DT_id $\} /$ Prescr $/\left\{P \_i d\right\} /$ Consent $/\left\{C_{-} i d\right\}$ & canonical \\
\hline 15 & /HospitalFilesManager/HospitalFile/\{HF_id\}/MedicalRecords/MedicalRecordsBox & canonical \\
\hline 16 & /MedicalRecordsManager/MedicalRecord//MR_id $\} /$ DailyTherapies/DailyTherapiesBox & canonical \\
\hline 17 & /MedicalRecordsManager/DailyTherapy//DT_id $\} / T r e a t m /\left\{T \_i d\right\} /$ ReportsBox & canonical \\
\hline
\end{tabular}




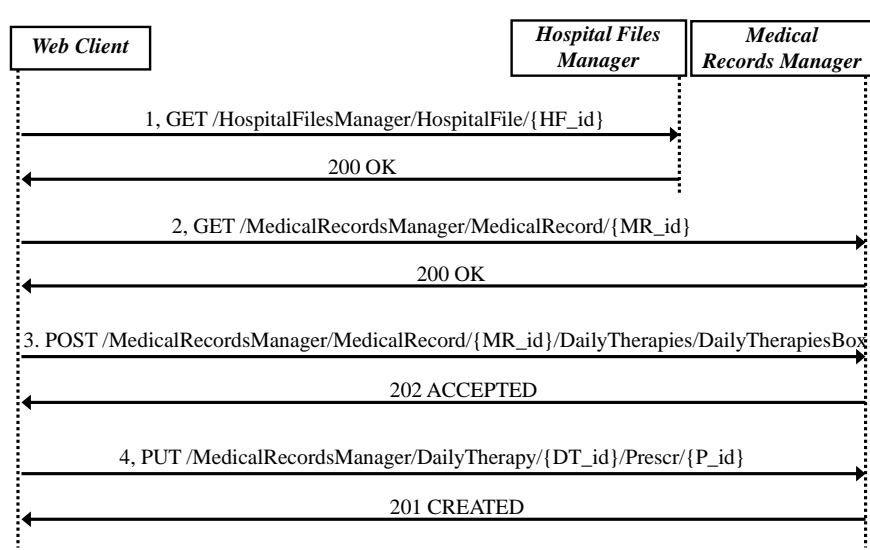

Fig. 5. Use case: creating a new drug prescription.

client performs an HTTP GET request to obtain the selected medical record. In the third step, the client performs an HTTP POST operation on the Daily Therapies Box URI to create a new Daily Therapy document (according to the model depicted in Figure 4). This is a composite operation (as shown in Figure 3 ) and is completely transparent to the client. Finally, in the fourth step, the client invokes an HTTP PUT operation on the previously created Daily Therapy and sends the drug prescription information provided by the physician in the request payload.

\section{PROTOTYPE IMPLEMENTATION}

This section describes the implementation of the PHARMA prototype. This prototype provides a basic implementation of the services mentioned in Section IV: Clinical Information retrieval, Medical record management, Medical report management and Daily Therapies management.

As shown in Figure 6, the PHARMA prototype consists of two main components:

- The IDN middleware that offers a uniform REST interface and a graph-based information model masking the heterogeneities of underlying legacy information systems.

- The PHARMA Web Application, which has been developed on top of the IDN middleware as an IDN-Compliant Application (IDN-CA).

The PHARMA web application exploits the information management capabilities provided by a distributed system of IDN-Nodes. Figure 6 shows a possible deployment architecture of PHARMA and InterDataNet (IDN) nodes. IDN offers capabilities to enable the asynchronous collaboration between distributed subjects. Exchanged data (e.g., personal health records, daily therapy) are structured in IDN-Documents and, according to IDN-IM specifications, are identified by HTTP URIs and accessed with HTTP verbs. The adoption of standard Web principles and technologies supports next extensions and the interoperability with external systems.

The PHARMA Web Application is made of client-side and server-side software modules, hereafter referred to as PHARMA Client and PHARMA Server, respectively.

\section{A. PHARMA Client}

The PHARMA Client implements the graphical user interface (GUI) for the end users (i.e., the health care staff). Therefore, it mainly consists of client-side presentation and content management logic. It has been implemented as a Mozilla Firefox extension by leveraging the XUL (XML User Interface Language) language [45] and JavaScript [46]. As a matter of fact, XUL and the Mozilla cross platform component object model (XPCOM) allow one to build rich cross-platform applications. Thanks to this programming framework, the PHARMA add-on is cross-platform, it is not bound to a specific Operative System and can run on heterogeneous devices (desktops, laptops and mobile devices). It is also possible to activate the off line mode and store application data on a local database for later synchronization.

Figure 7 provides a snapshot of the PHARMA Client showing the Daily Therapy form. Through this GUI, physicians can: 1) read information from patient's therapy; 2) create a new drug prescription (as described in section IV.C); 3) modify an existing prescription (e.g., changing the drug administration time); and 4) modify general information about the patient's Daily Therapy (e.g., adding a new documented patient's drug allergy).

\section{B. PHARMA Server}

The PHARMA Server is a web application that listens for HTTP requests coming from PHARMA Clients, and processes them by exploiting the underlying IDN REST APIs. In other words, when a PHARMA Server receives a Client request, it handles the request by translating it in a sequence of REST invocations to the proper IDN-Documents, processing the received outputs and building the response message to be returned to the originating PHARMA Client.

It is made up of two main components: the REST Controller, which interacts with the IDN middleware to retrieve or modify the information organized in IDN-Documents; and the XML binding layer, which manages the XML validation, marshaling and un-marshaling.

The PHARMA Server is implemented using the following technologies: the Spring framework [47], Java programming language [48], Java Architecture for XML Binding (JAXB2) [49] and Hibernate [50].

Basic security mechanisms have been implemented in the PHARMA Web Application to address confidentiality, integrity, authentication, authorization, and accounting

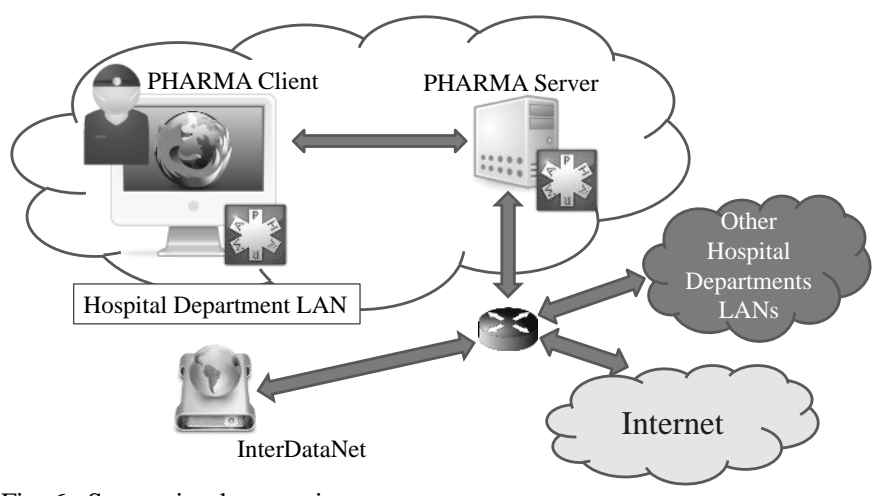

Fig. 6. System implementation. 
requirements. PHARMA Client users must be authenticated by the PHARMA Server to perform operations, while information exchange occurs through an HTTPS secured channel. In turn, PHARMA Servers must be authenticated by the IDN infrastructure to access the reserved resources. By definition, such a scenario requires several trust constraints between the actors. The data stored in IDN side are encrypted with an AES [51] algorithm. Critical actions such as a prescription creation require a strong accountability enforcement. To this purpose, the system includes a digital signature procedure designed on the basis of the Zhou-Gollman non-repudiation protocol [52].

\section{Implementation strategies}

PHARMA implementation strategy has been mainly influenced by the requirement of minimizing the size of response messages provided by the PHARMA Server. Indeed, two main design choices are possible: 1) the number of clients' requests are maximized to minimize the response payload. In this scenario a client makes several requests to get fine-grained information ready to be used; 2) the number of clients' requests are minimized and the size of transmitted data per request increases. In this scenario a client makes fewer requests accepting a wider amount of data per request.

Unfortunately, it is not possible to estimate the average HTTP requests number made by users due to their high variability depending on application contexts. It is possible to hypothesize that a practical worst case scenario is represented by a ward having twenty/twenty-five hospitalized patients and at the same time there are ten users accessing the system concurrently. A practical best case scenario might be the diagnostics ward where the workflows are "serialized": typically, the patients are examined one by one dropping the system load. Therefore, there is a high variance in the exchanged messages which makes it difficult to estimate the best strategy for a generic case.

We chose to minimize the amount of requests penalizing the size of the payload and delegating part of the computation to the client. This solution promotes system scalability, since the server has less information to process and the number of exchanged messages is limited.

\section{VALIDATION}

PHARMA's interface usability has been assessed so as to verify the concrete accessibility of the system. The assessment procedure referred to the IEC 62366 [53] specifications. The IEC 62366 standard specifies a process for validating usability as it relates to the safety of a medical device. It can be used to assess and mitigate risks caused by usability problems. The system's validation phase is thus extremely important since the objective of PHARMA is to reduce ADEs that may occur in drug prescription and administration processes.

The interface evaluation process consists of two main steps aimed at assessing different levels of usability:

1) evaluation of the possible interactions of stereotyped users, using a Cognitive Walkthrough methodology;

2) evaluation of the interface by usability and risk management experts, through a modified heuristic evaluation method.

A questionnaire has also been used, according to [54], but the preliminary results are still too weak to be considered in this work. Authors are considering performing a new campaign using validated questionnaires and a larger number of users.

Since each of these usability evaluation methods, taken alone,

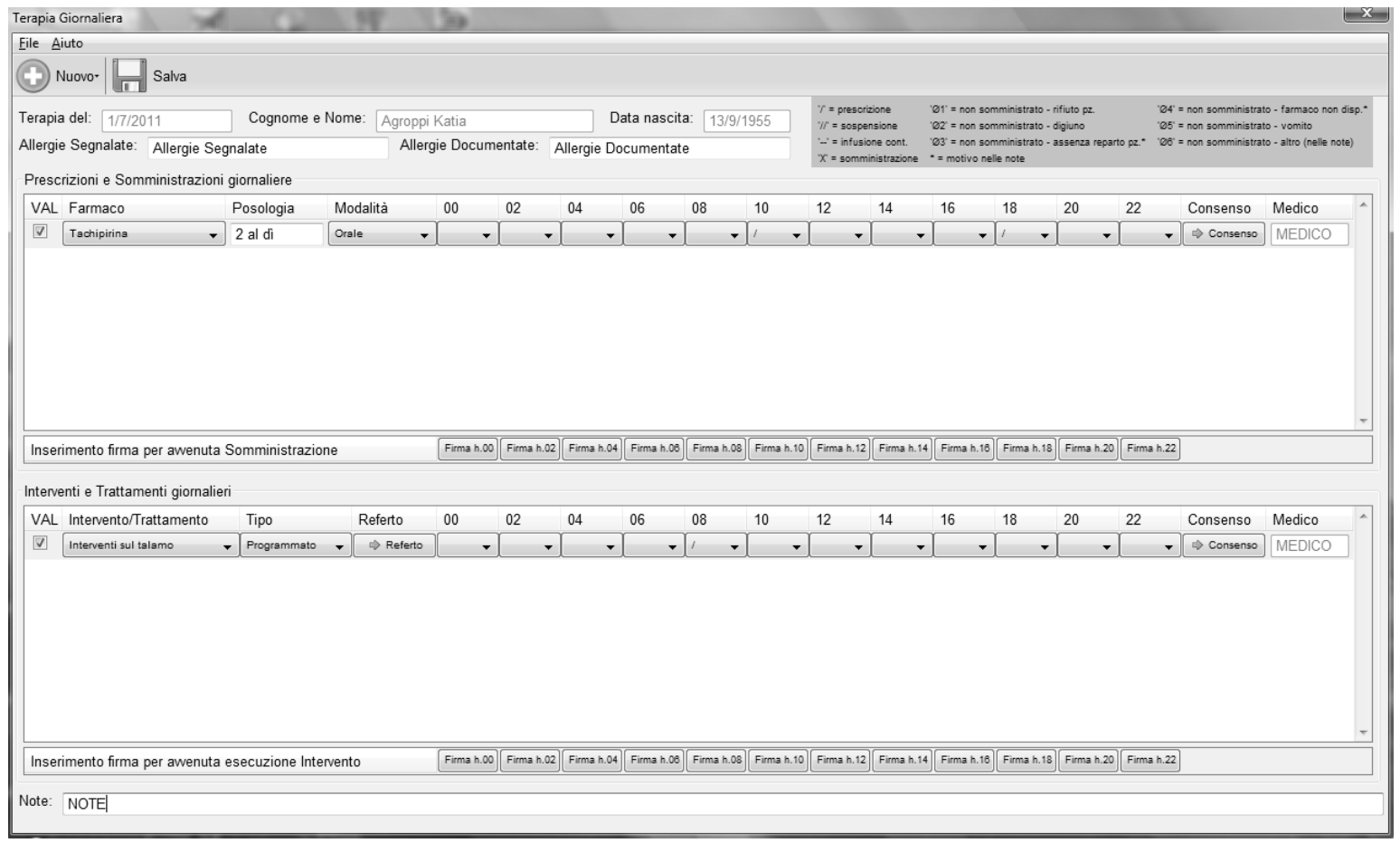

Fig. 7. A screenshot from the GUI. Displaying a specific daily therapy. 
shows limitations [55] (e.g. not revealing major missing functionality etc.), we decided to apply a combination of two different evaluation methods so as to rely on a more complete description of the system.

\section{A. Step 1: Cognitive Walkthrough}

The application of the Cognitive Walkthrough [56][57] method aims at evaluating the ease of use of the system. Given 1) a system description; 2) a description of a specific action that the user is requested to perform with the system; 3) a detailed tasks list required to perform such an action; and 4) the stereotype of the possible user (his/her knowledge, previous experience, etc.), the characteristics of the user-system interaction are assessed according to a defined set of guiding questions. The use case selected to perform the prototype GUI evaluation with the Cognitive Walkthrough method is "new drug prescription for a patient". In this use case, a biomedical engineer, using the PHARMA Client, executes the operations required to obtain the patient's daily therapy (as described in section IV.C). Ultimately, the biomedical engineer inserts the new prescription on the daily therapy window and he saves produced data in the daily therapy document. We chose a biomedical engineer since this is a figure that can merge both health care and technical competences.

This evaluation provides a preliminary assessment of the PHARMA prototype in a basic but significant use case. The tester managed to accomplish all the 6 operations that were necessary to complete the requested task (login, search for patient, open medical record, find the daily therapy, new prescription, save prescription). This means that the prototypal GUI is able to drive the user in his task. The tester reported that the system was able to give good feedback on the operations performed.

\section{B. Step 2: Heuristic evaluation}

A modified heuristic evaluation method [58] designed for medical devices was applied so as to identify usability problems and their severity. This heuristic evaluation refers to a class of techniques in which expert evaluators examine an interface for usability issues.

During a heuristic evaluation, experts walk through the interface and identify elements that violate usability heuristics. The usability heuristics for medical devices consist of 14 heuristics aimed at assessing: consistency; visibility of system state; match between system and world; minimalism; minimization of memory load; informative feedback; flexibility and efficiency; good error messages; preventing errors; clear closure; reversible actions; use of user's language; user control of the system; help and documentation. A five-level rating scale is adopted so as to rank the severity of the heuristic violation (from 0 , i.e. not a usability problem at all, to 4 i.e. usability catastrophe, imperative to fix before releasing the product). Two usability experts from the Risk Management Unit of the Tuscany Region evaluated our system according to this method. Each of them generated a list of usability problems which were evaluated according to the heuristics described in the method.
Once the two evaluators identified potential usability problems, the separate lists were compiled into a single master list in which the ratings from the individual evaluators were averaged and priorities assigned. Twelve usability problems have been identified and divided by priority into three sets: the low priority set contains four problems (mean severity rate 1), the average priority set contains five problems (mean severity rate 2), and the high priority set contains three problems (mean severity rate $3)$. Among the identified problems those labeled with the highest priority (mean severity rate 3 ) were mainly related to: i) Daily Therapies (ITCs) navigation, i.e., improving manageability and ease of browsing among ITCs (heuristics violated: flexibility and efficiency); ii) the displacement of objects within the ITC perspective, i.e., changing the prescription delete button position to avoid bad interpretations of the associated action (heuristics violated: reversible actions; minimalism); and iii) clarifying the procedure for inserting a new prescription (heuristics violated: clear closure). These usability problems, together with the risk mitigation modifications found with an FMECA process performed in two clinical departments (an Intensive Care Unit and an Internal Medicine Department) will drive the design process for the next release of PHARMA [59].

\section{CONCLUSIONS}

In this paper, we proposed PHARMA, a RESTful web information system for drug prescription, administration and registration. PHARMA allows the health care staff to access and share patient clinical data in a consistent and secure way.

The system has been designed according to the REST principles and Web standard protocols to favor integration with heterogeneous legacy systems and realized by leveraging the information modeling primitives and services provided by the InterDataNet middleware.

Specific efforts have been devoted to assess PHARMA's interface usability, with encouraging results. Analytic feedback of the heuristic evaluation process carried out by two usability experts of the Risk Management Unit of the Tuscany Region will be taken into account to ground future PHARMA improvements.

In the near future, we are determined to investigate the degree to which the introduction of the system introduces an extra learning load which impacts the healthcare staff workload. More specifically we would like to measure how closely the system fits the current personnel workflow.

Additionally, we will take into account a further integration of biomedical ontologies to enhance knowledge management, data integration and decision support [60]. We will consider the adoption of RxNorm [61][62], a controlled vocabulary of normalized names and codes for clinical drugs, and the Unified Medical Language System (UMLS) [63][64], a terminology integration system in which several ontologies are integrated. IDN can easily handle these elements. Indeed, references to such biomedical ontology concepts can be put into every IDN-represented medical resource metadata and managed 
through an IDN Semantic Search Service, under development.

Our main objective is to iteratively improve the functionalities of our system, so as to demonstrate a significant reduction in ADE, thanks to the introduction of PHARMA into the drug prescription and administration processes.

Moreover, PHARMA will be extended to integrate a Hospital Risk Management System [65] supporting the risk manager in assessing the quality of the medication service, and a Complex Event Processing (CEP) system [66] which will enable recognitions patterns of events identifying an abnormal use of the system.

Currently, security mechanisms have been implemented in the PHARMA Web Application. Research is underway to enforce security at an infrastructure level. The aim of this research activity is to enable content-centric security by exploiting and safeguarding the collaborative and content-centric nature of IDN in a global and federated domain.

Leveraging IDN capabilities, further research will address PHARMA integration with complementary systems to potentially reduce additional Adverse Drug Events including drug distribution errors, as detailed in [67].

\section{ACKNOWLEDGMENT}

The Authors thank Mr. Capannesi for his technical support. The Authors thank Dr. Chiarugi, Dr. Morelli, Dr. Cecatiello, Dr. Masi, Dr. Tartaglia, Dr. Tanzini, and Dr. Ranzani from the Tuscany Region Institute for interesting discussions and comments. The Authors thank Prof. Pirri for the fruitful discussions of all aspects of this work.

\section{REFERENCES}

[1] J.R. Nebeker, P. Barach, and M.H. Samore, "Clarifying Adverse Drug Events: A Clinician's Guide to Terminology, Documentation, and Reporting”, Ann Intern Med., vol. 140, pp. 795-801, 2004.

[2] S.C. Hartwig, S.D. Denger, and P.J. Schneider, "Severity-indexed, incident report-based medication error-reporting program", Springerlink, vol. 157, no. 9, pp. 2611-6, Dec. 1991.

[3] D.W. Bates, D.J. Cullen, N. Laird, L.A. Petersen, S.D. Small, D. Servi, G. Laffel, B.J. Sweitzer, B.F. Shea, R. Hallisey, M.V. Vliet, R. Nemeskal, and L.L. Leape, "Incidence of adverse drug events and potential adverse drug events: Implications for prevention", JAMA, vol. 274, pp. 29-34, Jul. 1995.

[4] Great Britain Department of Health, "An organisation with a memory: Report of an expert group on learning from adverse events in the NHS", London, The Stationery Office, 2000.

[5] P. Barach, and S.D. Small, "Reporting and preventing medical mishaps: lessons from non-medical near miss reporting systems", $B M J$, vol. 320, pp. 759-763, 2000.

[6] S. Aghazadeh, A.Q. Aliyev, and M. Ebrahimnejad, "The role of computerizing physician orders entry (CPOE) and implementing decision support system (CDSS) for decreasing medical errors", Application of Information and Communication Technologies (AICT), 5th International Conference on, pp.1-3, Oct. 12-14 2011.

[7] E. Ammenwerth, P. Schnell-Inderst, C. Machan, and U. Siebert, "The effect of electronic prescribing on medication errors and adverse drug events: a systematic review", JAMIA, vol. 15, pp. 585-600, 2008.

[8] Commissione Tecnica sul Rischio Clinico: "Risk Management in Sanità: il Problema degli Errori”, Rome, pp. 67-78, Mar. 2004.

[9] K. Shojania, B. Duncan, K. McDonald, and R.M. Wachter, "Making Health Care Safer: A Critical Analysis of Patient Safety Practices", Rockville, Md: Agency for Healthcare Research and Quality, 2001, ch. 6. Available: http://www.ahrq.gov/clinic/ptsafety.
[10] E. Iadanza, L. Baroncelli, A. Manetti, F. Dori, R. Miniati, G. B. Gentili, "An RFId smart container to perform drugs administration reducing adverse drugs events", IFMBE Proceedings 37, pp. 679-682, 2011.

[11] G.J. Kuperman, "Medication related clinical decision support in computerized provider order entry systems: A Review", JAMIA, vol. 14, pp. 29-40, Jan.-Feb. 2007.

[12] Health Information Systems, 2005. Available: http://www.hhs.gov/healthit/ahic.html

[13] M.H. Reckmann, J.I. Westbrook, Y. Koh, C. Lo, and R.O. Day, "Does computerized provider order entry reduce prescribing errors for hospital inpatients? A systematic review”, JAMIA, vol. 16, pp. 613-623, 2009.

[14] J.E. van Doormaal, P.M.L.A. van den Bemt, R.J. Zaal, A.C. Egberts, B.W. Lenderink, J.G. Kosterink, F.M. Haaijer-Ruskamp, and P.G. Mol, "The influence that electronic prescribing has on medication errors and preventable adverse drug events: an interrupted time-series study", JAMIA, vol. 16, pp. 816-825, 2009.

[15] R. Kaushal, K.G. Shojania, and D.W. Bates, "Effects of Computerized Physician Order Entry and Clinical Decision Support Systems on Medication Safety: A Systematic Review", Arch Intern Med., vol. 163, pp. 1409-1416, 2003.

[16] G.J Kuperman, and R.F. Gibson: "Computer Physician Order Entry: Benefits, Costs, and Issues", Annals of Internal Medicine, vol. 139, pp. 31-39, 2003.

[17] R. Koppel, J.P. Metlay, A. Cohen, B. Abaluck, A.R. Localio, S.E. Kimmel, and B.L. Strom, "Role of Computerized Physician Order Entry Systems in Facilitating Medication Errors", JAMA, vol. 293, pp. 1197-1203, 2005.

[18] G. Biffi Gentili, F. Dori, and E. Iadanza, "Dual-frequency active RFID solution for tracking patients in a children's hospital. Design method, test procedure, risk analysis, and technical solution", Proceedings of the IEEE, vol. 98, pp. 1656-1662, 2010.

[19] F. Bambi, I. Spitaleri, G. Verdolini, S. Gianassi, A. Perri, F. Dori, and E. Iadanza, "Analysis and management of the risks related to the collection, processing and distribution of peripheral blood haematopoietic stem cells", Blood Transfusion, vol. 7, pp. 3-17, 2009.

[20] Health Matrix. Available: http://www.healthmatrixcorp.com

[21] PatientKeeper. Available: http://www.patientkeeper.com

[22] G. Del Fiol, R.A. Rocha, R.L. Bradshaw, N.C. Hulse, and L.K. Roemer, "An XML model that enables the development of complex order sets by clinical experts", IEEE Trans. Inf. Technol. Biomed., vol. 9, no. 2, pp. 216-228, 2005

[23] S.H. Hsieh, I.C. Hou, P.H. Cheng, C.T. Tan, P.C. Shen, K.P. Hsu, S.L. Hsieh, and F. Lai, "Design and implementation of web-based mobile electronic medication administration record", J. Med. Syst, vol. 34, pp. 947-58, 2009.

[24] Health Level Seven International (HL7). Available: http://www.hl7.org/

[25] L. Bouzguenda, and M. Turki, "Coupling clinical decision support system with computerized prescriber order entry and their dynamic plugging in the medical workflow system", 2012 Int. Conf. on Inf. Technology and e-Services (ICITeS), pp.1-6, Mar. 24-26 2012.

[26] E.M. Campbell, D.F. Sittig, J.S. Ash, K.P. Guappone, and R.H. Dykstra, "Types of unintended consequences related to computerized provider order entry", JAMIA, vol. 13, pp. 547-56, 2006.

[27] J. Ash, P. Stavrl, and G. Kuperman, "A consensus statement on considerations for a successful CPOE implementation", JAMIA, vol. 10, pp. 229-34, 2003

[28] P.P. Sengstack, and B. Gugerty, "CPOE systems: success factors and implementation issues", Journal of healthcare information management, vol. 18, pp. 36-45, 2004.

[29] Physician Order Entry Team - Oregon Health \& Science University, "Computerized Physician/Provider Order Entry". Available: www.cpoe.org

[30] A. Ozdas, and R.A. Miller, "Care Provider Order Entry (CPOE): A Perspective on Factors Leading to Success or to Failure", Methods Inf Med, vol. 46, suppl. 1, pp. 128-137, 2007.

[31] M.C. Pettenati, S. Innocenti, D. Chini, D. Parlanti, and F. Pirri, "InterDataNet: A Data Web Foundation For The Semantic Web Vision", Iadis International Journal On Www/Internet, vol.6, issue 2, Dec. 2008.

[32] R.T. Fielding, "REST: Architectural Styles and the Design of Network-based Software Architectures", Doctoral dissertation, University of California, Irvine, 2000. 
[33] C. Pautasso, O. Zimmermann, and F. Leymann: "Restful web services vs. big web services: making the right architectural decision", In Proc. of the 17th Int. Conf. on World Wide Web (WWW'08), pp. 805-814, Apr. 2008.

[34] L. Richardson, and S. Ruby, "RESTful Web Services", O'Reilly \& Associates, May 2007.

[35] F. Paganelli, and D. Parlanti, "A Dynamic Composition and Stubless Invocation Approach for Information-Providing Services", IEEE Transactions on Network and Service Management, vol. 10, no. 2, pp. 218-230, Jun. 2013.

[36] M.C. Pettenati, L. Ciofi, F. Pirri, and D. Giuli, "Towards a RESTful Architecture for Managing a Global Distributed Interlinked Data/Content/Information-Space", Future Internet: Achievements and Promising Technology, Springer, vol. 6656, pp. 81-90, 2011.

[37] F. Paganelli, S. Turchi, L. Bianchi, L. Ciofi, M.C. Pettenati, F. Pirri, and D. Giuli, "An information-centric and REST-based approach for EPC Information Services", Journal of Communications Software and Systems, vol. 9, no. 1, pp. 14-23, Mar. 2013

[38] R.H. Sprague Jr., "Electronic Document Management: Challenges and Opportunities for Information Systems Managers", MIS Quarterly, vol. 19, no. 1, pp. 29-49, Mar. 1995.

[39] T. Bellandi, F. Ranzani, and F. Draicchio, "Ergonomics Applied to Adverse Drug Events Prevention in Acute Care Hospitals: The Integrated Therapeutic Chart", Proceedings IEA 2006 Congress, Elsevier, 2006.

[40] Center for Clinical Risk Management and Patient Safety (GRC): "STU Scheda Terapeutica Unica", 2006. Available: http://www.regione.toscana.it/regione/multimedia/RT/documents/2010/ 09/20/1284967185329_scheda\%20terapeutica\%20unica.pdf

[41] International Classification of Diseases (ICD). Available: http://www.who.int/classifications/icd/en/

[42] Object Management Group: "BPMN Business Process Modeling Notation". Available: http://www.bpmn.org/

[43] BizAgi: "BPMN Process Modeler Software". Available: http://www.bizagi.com/

[44] L. Bianchi: "PHARMA (Personal HospitalizAtion Records MAnagement): REST Telematics System for the aggregation and reuse of personal Health data on the Web", M.S. thesis, Dept. Electron. and Telecomm. Eng., University of Florence, Florence, Italy, 2011. Available: http://www.interdatanet.org/wiki/index.php/File:Tesi_Bianchi.pdf

[45] XML User Interface Language. Available: https://developer.mozilla.org/en-US/docs/XUL

[46] Standard ECMA-262 ECMAScript Language Specification. Available: http://www.ecma-international.org/publications/standards/Ecma-262.ht $\mathrm{m}$

[47] SpringSource. Spring.net. Available: http://www.springframework.net/

[48] T. Lindholm, and F. Yellin, "Java virtual machine specification", Boston (MA), Addison-Wesley Longman Publishing Co., Inc., 1999.

[49] GlassFish Community. Jaxb reference implementation. Available: http://jaxb.java.net/

[50] JBoss Community. Hibernate. Available: http://www.hibernate.org/

[51] J. Daemen, and V. Rijmen, "The design of Rijndael: AES-the advanced encryption standard". Heidelberg, Springer, 2002.

[52] K. Kwangjo, P. Sangjoon, and B. Joonsang, "Improving fairness and privacy of Zhou-Gollmann's fair non-repudiation protocol", Proceedings, International Workshops on, pp.140-145, 1999.

[53] IEC 62366:2007 Medical devices - Application of usability engineering to medical devices.

[54] A. Karahoca, E. Bayraktar, E. Tatoglu, and D. Karahoca, "Information system design for a hospital emergency department: A usability analysis of software prototypes", Journal of Biomedical Informatics, vol. 43, issue 2, pp. 224-232, Apr. 2010

[55] J. Nielsen, "Usability Engineering", Boston (MA), AP Professional, 1993.

[56] C. Wharton, J. Rieman, C. Lewis, and P. Polson, "The cognitive walkthrough method: a practitioner's guide", New York (NY), John Wiley \& Sons, Inc., 1994, pp. 105-140.

[57] J. Nielsen, and R.L. Mack, "Usability Inspection Methods". New York (NY), John Wiley \& Sons, Inc., 1994.

[58] J. Zhang, T.R. Johnson, V.L. Patel, D.L. Paige, and T. Kubose, "Using usability heuristics to evaluate patient safety of medical devices", Journal of Biomedical Informatics, vol. 36, pp. 23-30, 2003.

[59] E. Iadanza, F. Gaudio, and F. Marini, "The diagnostic-therapeutic process. Workflow analysis and risk management with IT tools", Proc. of the 35th Annual Int. Conf. of the IEEE Engineering in Medicine and Biology Society (EMBC13), Osaka, Japan, Jul. 3-7 2013, in press.

[60] O. Bodenreider, "Biomedical ontologies in action: role in knowledge management, data integration and decision support", Yearb Med Inform 47, pp. 67-79, 2008.

[61] S. Liu, M. Wei, R. Moore, V. Ganesan, and S. Nelson, "RxNorm: prescription for electronic drug information exchange", IT Professional, vol. 7, pp. 17-23, 2005

[62] RxNorm. Available: http://www.nlm.nih.gov/research/umls/rxnorm/

[63] O. Bodenreider, "The unified medical language system (UMLS): integrating biomedical terminology", Nucleic Acids Research 32, pp. 267-270, 2004

[64] Unified Medical Language System (UMLS). Available: http://www.nlm.nih.gov/research/umls/

[65] G. Bernardini, F. Paganelli, M. Manetti, A. Fantechi, and E. Iadanza, "SYRMA: a Tool for a System Approach to Risk Management in Mission Critical Systems", International Journal of Business Information Systems, vol. 13, no. 1, pp. 21-44, 2013.

[66] I. Zappia, F. Paganelli, and D. Parlanti, "A Lightweight and Extensible Complex Event Processing System for Sense and Respond Applications", Expert Systems with Applications, vol. 39, no. 12, pp. 10408-10419, 2012.

[67] E. Iadanza, M.C. Pettenati, L. Bianchi, S. Turchi, L. Ciofi, F. Pirri, G. Biffi Gentili, and D. Giuli, "Telematics integrated system to perform drugs prescription and administration reducing adverse drug events", Engineering in Medicine and Biology Society (EMBC), 2012 Annual Int. Conf. of the IEEE, pp.6082-6085, Aug. 28 2012-Sep. 12012.

L. Bianchi received the "Laurea" (M.S.) degree (cum laude) in Computer Engineering, from the University of Florence, Italy, in 2011. Since January 2012 he is Ph.D. student in "Informatics, Systems and Telecommunications". $\mathrm{He}$ is presently with the Telematic Systems and Remote Sensing group at the Department of Electronic Engineering, University of Florence.

F. Paganelli (M'07) received a Ph.D. degree in Telematics and Information Society from the University of Florence, Italy, in 2004. She is a Senior Researcher at the National Interuniversity Consortium for Telecommunications (CNIT), Italy. Her research interests include context-aware systems, service-oriented computing and communication, and next generation networks.

Maria Chiara Pettenati is Program Manager at ICON (International Center of Computational Neurophotonics) Foundation. She received the M.S. degree in electronics engineering in 1996. In 2000 she received the Ph.D. title in Telematics and Information Society. Until December 2011 she was researcher at the Electronics and Telecommunications Dept. of the University of Florence.

Stefano Turchi graduated in Computer Engineering (M.S.) in 2010 at the University of Florence. From January 2011 he is a Ph.D. Student in "Informatics, Systems and Telecommunications" Doctoral Course. Currently he is researching in the field of Distributed Architectures, Web Applications, Data Security and Data Privacy in distributed and granular data scenarios.

Lucia Ciofi obtained a Ph.D. in Telematics and Information Society at the University of Florence in 2011. Since 2012 she holds a post-doctoral research position in the Department of Electronics and Telecommunications of the University of Florence. Her main research interests are: Web Science, REST, Cloud Computing.

Dino Giuli (SM'84) is Full Professor in Telecommunications at the Department of Electronics and Telecommunications of the University of Florence. Since 1996 he is Promoter and Scientific Coordinator of the Ph.D. program in "Telematics and Information Society". His research activities are focused in the domain of Telematics and Environmental Monitoring Systems. $\mathrm{He}$ is AEI member and IEEE senior member.

Ernesto Iadanza (M'07) received a Ph.D. degree in Telematics and Information Society at the University of Florence, Florence, Italy, in 2013. He is currently Adjunct Professor of Clinical Engineering and Biomedical Instrumentation at the University of Florence. His main research fields are healthcare engineering, clinical engineering, and risk management in healthcare. 